\title{
Immobilizing Au Nanoparticles with Polymer Single Crystals, Patterning and Asymmetric Functionalization
}

\author{
Bing Li and Christopher Y. Li
}

\begin{abstract}
A. J. Drexel Nanotechnology Institute and Department of Materials Science and Engineering, Drexel University, Philadelphia, PA 19104
\end{abstract}

\section{Supporting Information}

\section{Materials:}

Thiol-terminated polyethylene oxide (HS-PEO, $2 \mathrm{k}$ and $48.5 \mathrm{k} \mathrm{g} / \mathrm{mol}$ ) was purchased from Polymersource Inc. Amyl acetate, hexanedithiol, gold sol (5 nm and $20 \mathrm{~nm}$ ), tetraoctylammonium bromide (TOAB), sodium oleate, hexane, magnesium chloride and molecular seize were purchased from Aldrich. All materials were used as received.

\section{Experimental details:}

Preparation of HS-PEO single crystals using the self-seeding method:

$1.2 \mathrm{mg}$ HS-PEO (2k) was dissolved in $3 \mathrm{~g}$ amyl acetate at $60{ }^{\circ} \mathrm{C}$ for $10 \mathrm{~min}$. Then the solution was quenched to $5{ }^{\circ} \mathrm{C}$ for $3 \mathrm{hrs}$. The resulted crystals were partially dissolved at $44{ }^{\circ} \mathrm{C}$ for $10 \mathrm{~min}$ to obtain the crystal seeds. The solution was then brought to $24{ }^{\circ} \mathrm{C}$ and crystallized for 1 day. The suspension of the single crystals was isothermally filtered to remove uncrystallized polymers.

$1.0 \mathrm{mg}$ HS-PEO (48.5k) was dissolved in $1 \mathrm{~g}$ amyl acetate at $60{ }^{\circ} \mathrm{C}$ for $10 \mathrm{~min}$. Then the solution was quenched to $5{ }^{\circ} \mathrm{C}$ for 3 hrs. The resulted crystals were partially dissolved at $48{ }^{\circ} \mathrm{C}$ for 10 min to get the crystal seeds. Finally, the solution was brought to $38{ }^{\circ} \mathrm{C}$ and crystallized for 2 hrs. The suspension of the single crystals was isothermally filtered to remove uncrystallized polymers.

Immobilization of 5nm AuNPs on HS-PEO single crystals:

$1 \mathrm{~g}$ of $1 \%$ TOAB/amyl acetate was mixed with $1 \mathrm{~g}$ gold sol $(5 \mathrm{~nm})$. The mixture was sonicated for $1 \mathrm{~min}$ and centrifuged for $10 \mathrm{~min}$ at $2000 \mathrm{rpm}$. The amyl acetate phase was collected. Molecular seize was used to remove the residue water. HS-PEO single crystal suspension was drop-cast on carbon-coated TEM grids. These grids were immersed in the TOAB-protected AuNPs dispersed in amyl acetate for 2 hrs. Amyl acetate was used to wash out the free AuNPs on the grids after the incubation. 
Preparation of bilayer AuNP/PEO hybrids:

AuNP-coated HS-PEO single crystals obtained from the previous step were incubated in $1 \%$ Hexanedithiol/amyl acetate solution for 2 hrs. The samples were washed with amyl acetate before being immersed in gold sol $(20 \mathrm{~nm})$ for 2 hrs. Amyl acetate was used to wash out the free AuNPs after incubation.

Synthesis of free binary AuNP complexes via bilayer AuNP/PEO hybrids:

Following the same procedure as described in preparation of bilayer AuNP/PEO hybrids except replacing TEM grids with small pieces of glass. The final product was placed in ethanol. Sonication was used to take the single crystals off the glass and break the bilayer AuNP/PEO hybrids. After sonicating the sample for half an hour, one drop of the sample was placed on a carbon-coated Ni grid for TEM observation. $1 \mathrm{ml}$ sample was transferred to a cuveette for UV-Vis measurement.

\section{Immobilization of 20nm AuNPs on HS-PEO single crystals:}

$10 \mathrm{ml}$ hexane and $10 \mathrm{mg}$ sodium oleate were added to $10 \mathrm{~g} 20 \mathrm{~nm}$ gold colloid. The mixture was emulsified by vigorous stirring at room temperature for 2 hrs. $0.12 \mathrm{~g}$ magnesium chloride in $1.5 \mathrm{ml}$ water was then added with stirring. On standing for $4 \mathrm{hrs,}$ the mixture was transformed from an emulsion into two liquid phases. The hexane phase changed into pink while water phase became colorless, indicating AuNPs was transferred into hexane. The hexane phase was collected. Molecular seize was used to remove the residue water. HS-PEO single crystal suspension was drop-cast on a cover glass, which was immersed in the gold/hexane colloid for $1 \mathrm{hr}$. Hexane was used to wash out the free AuNPs after incubation.

Synthesis of free binary AuNPcomplexes via asymmetrically functionalized $20 \mathrm{~nm}$ AuNP precursors

The $20 \mathrm{~nm}$ AuNP-coated HS-PEO single crystals obtained from the previous step were incubated in $1 \%$ Hexanedithiol/amyl acetate solution for $30 \mathrm{~min}$. The samples were washed by amyl acetate thoroughly before being placed in $2 \mathrm{~g}$ water. After sonication for 0.5 hrs, free asymmetrically functionalized AuNPs, which have PEO on one side and hexanedithiol on the other, were dispersed in water. $1 \mathrm{~g} 5 \mathrm{~nm}$ gold colloid was then mixed with the obtained asymmetrically functionalized AuNPs and the reaction was allowed to take place overnight. One drop of the sample was placed on a carbon-coated Ni grid for TEM observation. 


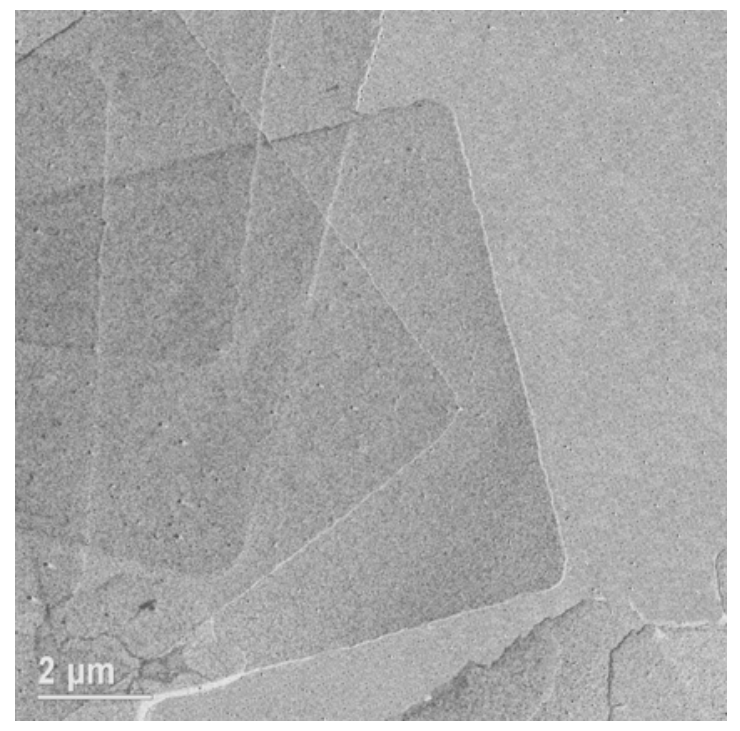

Figure S. 1. HS-PEO (2k) single crystals formed by solution crystallization.

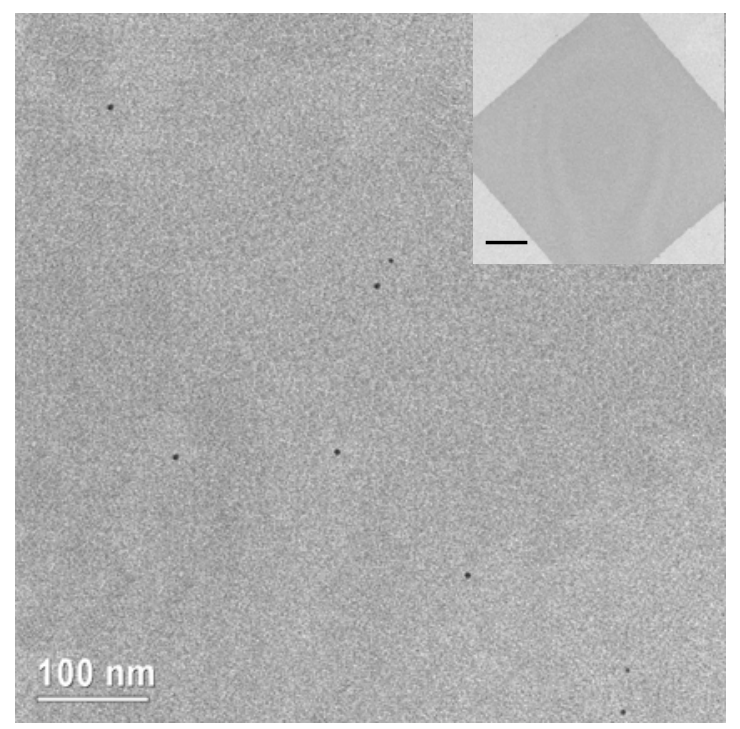

Figure S. 2. AuNPs on the surface of HO-PEO (2k) single crystals after incubation (inset shows the entire single crystal, scale bar $2 \mu \mathrm{m}$ ). Only a few AuNPs can be seen on the crystal surface due to the weak Van der Waal interaction. 


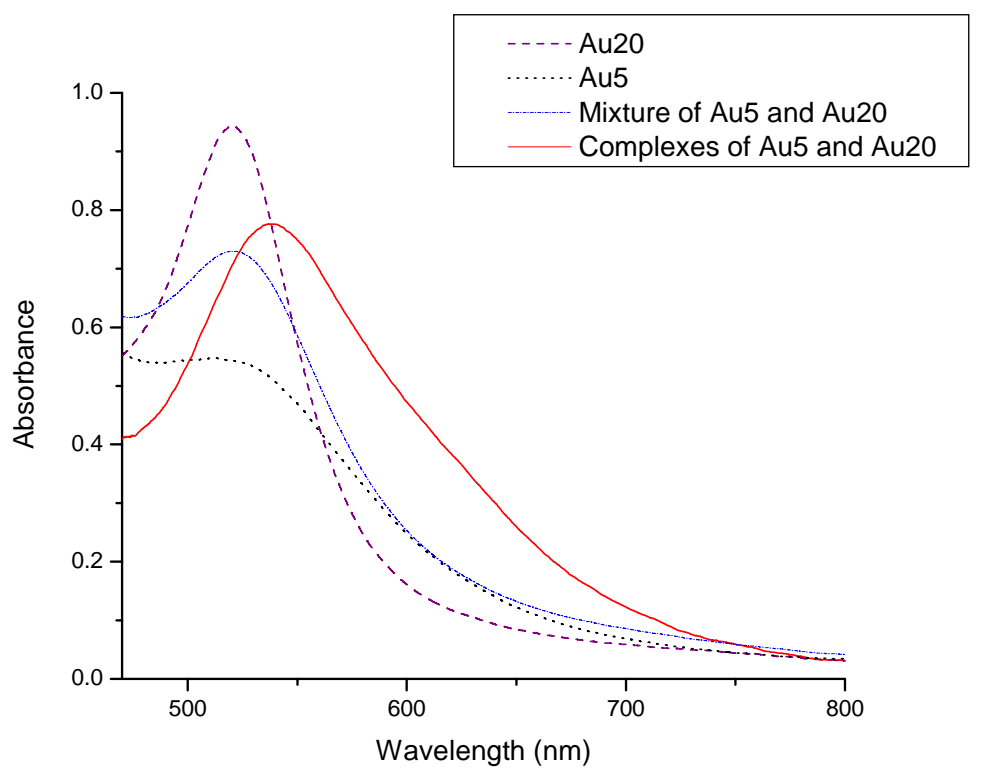

Figure S. 3. UV-Vis spectra of various gold colloids. Au5 and Au20 stand for $5 \mathrm{~nm}$ AuNPs and $20 \mathrm{~nm}$ AuNPs, respectively. The surface plasmon band (SPB) of $20 \mathrm{~nm}$ AuNPs undergoes red-shift from $520 \mathrm{~nm}$ to $537 \mathrm{~nm}$ after forming binary AuNP complexes with $5 \mathrm{~nm}$ AuNPs. Detailed investigation on the ligand molecular length effect on the SPB shifting is currently under investigation.

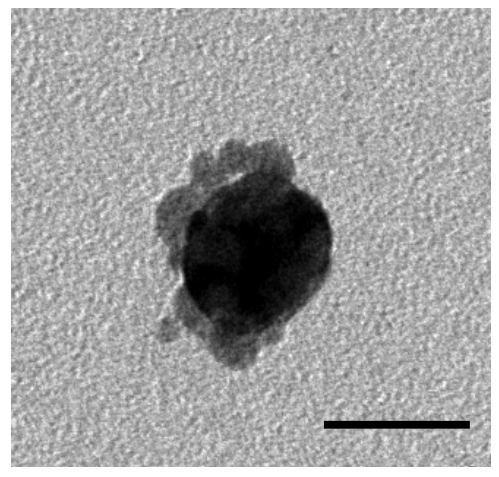

Figure S. 4. Multi-particle complexes formed by mixing asymmetrically functionalized $20 \mathrm{~nm}$ AuNPs with $5 \mathrm{~nm}$ AuNPs. Scale bar: $20 \mathrm{~nm}$. 